\title{
Naringin ameliorates endothelial dysfunction in fructose-fed rats
}

\author{
WACHIRAWADEE MALAKUL ${ }^{1}$, SIRINAT PENGNET ${ }^{2}$, CHANON KUMCHOOM $^{1}$ and SAKARA TUNSOPHON ${ }^{1}$ \\ ${ }^{1}$ Department of Physiology, Faculty of Medical Science, Naresuan University, Phitsanulok 65000; \\ ${ }^{2}$ Division of Physiology, School of Medical Science, University of Phayao, Phayao 56000, Thailand
}

Received October 6, 2017; Accepted January 8, 2018

DOI: $10.3892 /$ etm.2018.5759

\begin{abstract}
High fructose consumption is associated with metabolic disorders including hyperglycemia and dyslipidemia, in addition to endothelial dysfunction. Naringin, a flavonoid present in citrus fruit, has been reported to exhibit lipid lowering, antioxidant, and cardiovascular protective properties. Therefore, the present study investigated the effect of naringin on fructose-induced endothelial dysfunction in rats and its underlying mechanisms. Male Sprague-Dawley rats were given $10 \%$ fructose in drinking water for 12 weeks, whereas control rats were fed drinking water alone. Naringin $(100 \mathrm{mg} / \mathrm{kg})$ was orally administered to fructose fed rats during the last 4 weeks of the study. Following 12 weeks, blood samples were collected for measurement of blood glucose, serum lipid profile and total nitrate/nitrite (NOx). Vascular function was assessed by isometric tension recording. Aortic expression of endothelial nitric oxide synthase (eNOS), phosphorylated eNOS (p-eNOS), and nitrotyrosine were evaluated by western blot analysis. Fructose feeding induced increased levels of blood glucose, total cholesterol, triglyceride, and low density lipoprotein. In rat aortae, fructose reduced acethycholine-induced vasorelaxation, without affecting sodium nitroprusside-induced vasorelaxation. Treatment of fructose-fed rats with naringin restored fructose-induced metabolic alterations and endothelial dysfunction. Fructose-fed rats also exhibited decreased serum NOx level, reduced eNOS and p-eNOS protein expression, and enhanced nitrotyrosine expression in aortae. These alterations were improved by naringin treatment. The results of the present study suggested that naringin treatment preserves
\end{abstract}

Correspondence to: Dr Wachirawadee Malakul, Department of Physiology, Faculty of Medical Science, Naresuan University, 99 Moo 9 Phitsanulok-Nakhonsawan Road, Muang, Phitsanulok 65000, Thailand

E-mail:wachirawadeem@hotmail.com

Abbreviations: TC, total cholesterol; TG, triglyceride; HDL-C, high density lipoprotein cholesterol; LDL-C, low density lipoprotein cholesterol; NO, nitric oxide; eNOS, endothelial nitric oxide synthase; ACh, acethycholine; SNP, sodium nitroprusside; PE, phenylephrine; NOx, nitrate/nitrite

Key words: naringin, fructose-fed rats, endothelial dysfunction, nitric oxide, nitrotyrosine endothelium-dependent relaxation in aortae from fructose fed rats. This effect is primarily mediated through an enhanced NO bioavailability via increased eNOS activity and decreased NO inactivated to peroxynitrite in aortae.

\section{Introduction}

Fructose is widely used as a sweetener in many processed foods and beverages. The consumption of processed foods and beverages has increased dramatically over the past few decades. There is increasing evidence that high dietary fructose consumption causes dyslipidemia, insulin resistance, obesity, and endothelial dysfunction $(1,2)$. All of these factors are associated with increased risk of cardiovascular diseases (CVD), one of the main cause of morbidity and mortality worldwide $(3,4)$.

Endothelial dysfunction is considered one of the initial stages in the development of atherosclerosis and CVD, and a crucial target for the prevention of CVD (5). Vascular endothelium plays an important role in modulating vascular tone through the synthesis and release of several vasoactive factors including nitric oxide (NO). NO is generated from the conversion of L-arginine to L-citrulline by endothelial NO synthase (eNOS) and has a potent vasodilator, anti-inflammatory, and antithrombotic properties (6). Reduction of NO bioavailability, caused by reduced eNOS activity and/or accelerated NO degradation, results in impaired endothelium-dependent vasorelaxation, increased thrombus formation, and progressive atherogenesis (7). There is growing evidence that fructose fed animals exhibited impaired endothelium-dependent relaxation (8-11) and decreased NO bioavailability (12-14). High fructose consumption is also reported to induce oxidative and nitrative stress in vascular tissues and that oxidative/nitrative stress seems to play a major role in endothelial dysfunction. Indeed, elevated levels of reactive oxygen species (ROS) cause a decrease in bioavailability of $\mathrm{NO}$ and an increase in production of powerful oxidant peroxynitrite that induces eNOS inactivation $(15,16)$. A number of studies reported that decreased eNOS expression and increased nitrotyrosine expression were found in aortae from fructose fed rats $(8,17,18)$. Therefore, the increased oxidative stress and the decreased NO synthesis have been proposed to be involved in high fructose-induced endothelial dysfunction.

Naringin (4',5,7-trihydroxyflavone 7-rhamnoglucoside) is one of the major constituents of the flavonoids in citrus fruit, especially grapefruit (19). Naringin and its colonic metabolite, 
naringenin, have been reported to possess antihyperglycemic, anti-inflammatory, anti-oxidative, and antihyperlipidemic effects (19-24). Moreover, naringin has been shown to exhibit cardiovascular protective effects in animal models by preserving endothelial function, enhancing NO bioavailability, and reducing oxidative stress $(21,25)$. Based on these findings, we hypothesized that naringin may improve endothelial dysfunction through its modulation of NO bioavailabity and oxidative stress. However, to our knowledge, the effect of naringin on high fructose-induced endothelial dysfunction has never been reported. Therefore, the aim of this study was to determine whether naringin could attenuate endothelial dysfunction in fructose-fed rats and to elucidate the mechanism underlying the alleviation of endothelial dysfunction.

\section{Materials and methods}

Chemicals. Naringin, acethycholine (ACh), $N^{\mathrm{G}}$-nitroL-arginine, indomethacin, phenylephrine (PE), and sodium nitroprusside were purchased from Sigma-Aldrich (Merck KGaA, Darmstadt, Germany), and a nitrate/nitrite (NOx) assay kit was purchased from Cayman Chemical Company, (Ann Arbor, MI, U). Antibodies against eNOS, phosphorylated eNOS at Ser1177 and $\beta$-actin were obtained from Cell Signaling Technology, Inc. (Danvers, MA, USA), and the antibody against nitrotyrosine and HRP-conjugated anti-rabbit IgG were from Merck KGaA. Fructose was obtained from Charoentavorn Supply Co. (Samutprakarn, Thailand).

Animals and experimental design. Male Sprague-Dawley (SD) rats weighing 180-200 g were obtained from the National Laboratory Animal Center, Mahidol University, Bangkok, Thailand. All animals used in this study were housed at a constant temperature of $20-22^{\circ} \mathrm{C}$, with a $12 \mathrm{~h}$ light-dark cycle and allowed free access to tap water and the standard rat feed for one week. After acclimatization, the rats were randomly divided into three experimental groups: Group I, the control group (C) which received normal drinking water; Group II, the fructose group $(\mathrm{F})$ which received fructose solution and the vehicle $0.1 \%$ carboxymethylcellulose (CMC); and Group III, the fructose plus naringin group $(\mathrm{FN})$, which received fructose solution and naringin (100 $\mathrm{mg} / \mathrm{kg} /$ day) suspended in $0.1 \%$ $\mathrm{CMC}$. The fructose solution was prepared as $10 \%$ fructose $(w / v)$ in the drinking water and administered every day to the rats for 12 weeks ad libitum. Naringin (100 mg/kg/day) or the vehicle $0.1 \% \mathrm{CMC}$ was administered daily by oral gavage for the last 4 weeks of fructose feeding. The concentration of naringin used in the present study was selected according to previous studies using animal models which indicated that this dosage exerted improving vascular dysfunction (21).

All animal procedures were performed within the institutional guidelines for the care and use of laboratory animals and were approved by the Animal Ethics Committee of Naresuan University, Thailand (approval number: 57040023).

Preparation of serum and aortic tissues. At the end of 12 weeks of fructose feeding, overnight fasting rats were anesthetized with an intraperitoneal injection of pentobarbital $(50 \mathrm{mg} / \mathrm{kg})$ and blood samples were collected by cardiac puncture. Blood was then centrifuged at $1,500 \mathrm{xg}$ for $15 \mathrm{~min}$ at $4^{\circ} \mathrm{C}$ to obtain serum, which was stored at $-20^{\circ} \mathrm{C}$ until further analysis. The descending thoracic rat aorta was isolated and divided into two parts: One part was placed in Krebs bicarbonate solution [composition (mM): $\mathrm{NaCl} 118.0, \mathrm{NaH}_{2} \mathrm{CO}_{3}$ 25.0, glucose 11.0, $\mathrm{CaCl}_{2}$ 1.6, $\mathrm{KCl} 4.7, \mathrm{KH}_{2} \mathrm{PO}_{4} 1.2$ and $\mathrm{MgSO}_{4}$ 1.18] for determination of vasorelaxant response, and the other part was frozen by liquid nitrogen and stored at $-80^{\circ} \mathrm{C}$ until used for western blot analysis.

Biochemical analysis. At the end of experimental period, blood glucose levels were measured using an Accu-Check glucometer (Roche Diagnostics GmbH, Mannheim, Germany). Serum concentrations of total cholesterol (TC), triglyceride (TG), and high density lipoprotein cholesterol (HDL-C) were measured colorimetrically with commercial kits (Human Company, Wiesbaden, Germany). The serum low density lipoprotein cholesterol (LDL-C) level was calculated using the formula of Friedewald: LDL=TC-HDL-(TG/5) (26). The serum levels of NOx, the final products of NO metabolism, were determined by using NOx fluorometric assay kit (Cayman Chemical Company).

Measurement of vascular reactivity in the thoracic aorta. As previously described (27), after the removal of superficial connective tissue and fat surrounding the aorta, the isolated thoracic aorta was cut into rings (3-4 $\mathrm{mm}$ in length) and mounted on stainless steel hooks in an organ bath chamber containing oxygenated Krebs bicarbonate solution $\left(95 \% \mathrm{O}_{2}\right.$ and $\left.5 \% \mathrm{CO}_{2}\right)$ maintained at $37^{\circ} \mathrm{C}$. The isometric tension of aortic ring was recorded using a force transducer (model no. FT03, Grass Medical Instruments) connected to a Powerlab Data Acquisition System (AD Instruments, Sydney, Australia). Before starting the experiment, the resting tension of each ring was adjusted to $1 \mathrm{~g}$ and equilibrated for $60 \mathrm{~min}$. Each aortic ring was maximally contracted with an isotonic, high potassium salt solution (KPSS, $123 \mathrm{mM}$ ). To investigate the effect of the treatment on the relaxant responses of the aortic rings, the endothelium-dependent relaxation to ACh $\left(10^{-9}-10^{-5}\right.$ $\mathrm{M})$ and the endothelium-independent relaxation to sodium nitroprusside (SNP, $10^{-9}-10^{-5} \mathrm{M}$ ) were tested on the aortic rings precontracted submaximally with $\mathrm{PE}\left(10^{-9}-10^{-6} \mathrm{M}\right)$. All changes in the tension were expressed as a percentage of the precontraction. To assess the contribution of the endothelial vasodilator factors in response to $\mathrm{ACh}$, concentration-response curves to $\mathrm{ACh}$ were performed in the absence or presence of the NO synthase (NOS) inhibitor $N^{\mathrm{G}}$-nitro-L-arginine (L-NNA; $100 \mathrm{mmol} / \mathrm{l})$, the cyclo-oxygenase inhibitor indomethacin (10 mmol/l), or L-NNA plus indomethacin.

Western blot analysis of eNOS, p-eNOS and nitrotyrosine. Aortic tissue samples were homogenized in ice-cold RIPA buffer containing protease and phosphatase inhibitor and centrifuged at $15,000 \mathrm{x} \mathrm{g}$ for $20 \mathrm{~min}$ at $4^{\circ} \mathrm{C}$. The supernatants were collected and the protein concentrations were measured by the bicinchoninic acid protein assay kit (Merck KGaA). Each sample of aortic homogenates (40 $\mu \mathrm{g}$ protein) was separated by $7.5 \%$ sodium dodecyl sulfate-polyacrylamide gel electrophoresis and then transferred to polyvinylidenedifluoride membranes which were blocked with 5\% nonfat dry milk for $1 \mathrm{~h}$ and then incubated with anti-eNOS (1:500), 
anti-phospho-eNOSSer1177 (1:500), anti-nitrotyrosine $(1: 1,000)$ and anti- $\beta$ actin $(1: 5,000)$ at $4^{\circ} \mathrm{C}$ overnight. The membranes were then washed with tris-buffered saline with Tween-20 (TBST) and incubated with anti-rabbit horseradish peroxidase conjugated secondary antibody $(1: 5,000)$ at room temperature for $1 \mathrm{~h}$. Protein expression was visualized using Luminata forte HRP detection reagent (Merck KGaA). Protein bands were quantified by densitometry using a Bio-Rad image analysis system (Quantity One; Bio-Rad Laboratories, Inc., Hercules, CA, USA) and normalized to $\beta$-actin protein expression.

Statistical analysis. The results are expressed as the mean \pm SEM. Relaxation responses to ACh or SNP were expressed as a percentage of PE induced precontraction. Concentration-response to agonists were fitted to a sigmoidal curve using GraphPad Prism, version 5 (GraphPad Software Inc., San Diego, CA, USA) to calculate the sensitivity of each agonist $\left(\mathrm{pEC}_{50}\right)$. Maximum relaxation $\left(\mathrm{R}_{\max }\right)$ to $\mathrm{ACh}$ or SNP was calculated as a percentage of precontraction to PE. The $\mathrm{pEC}_{50}$ and $\mathrm{R}_{\max }$ values were compared among groups using one-way analysis of variance (ANOVA) with post hoc multiple comparisons using Newman-Keuls or Dunnett's test (GraphPad Software Inc.). P $<0.05$ was considered to indicate a statistically significant difference.

\section{Results}

Effect on body weight and metabolic parameters. As shown in Table I, there was no difference in body weight gain among the three experimental groups by the end of the experiment. The rats that had consumed fructose in drinking water for 12 weeks showed significantly increased levels of blood glucose, TC, TG and LDL-C, compared to control rats. Naringin $(100 \mathrm{mg} / \mathrm{kg} /$ day $)$ treatment for 4 weeks significantly attenuated fructose-induced metabolic changes in rats (Table I). These results indicate that naringin improved metabolic abnormalities in fructose fed rats including hyperglycemia and hyperlipidemia.

Effect on endothelial function. Endothelium-dependent and independent relaxation to ACh and SNP respectively, are shown in Fig. 1 and Table II. Fructose feeding significantly decreased the maximum relaxation but not the sensitivity to $\mathrm{ACh}$ in aortae compared to the control rats, indicating that fructose impaires endothelial function. Relaxation responses to SNP were not significantly different between the control and fructose fed rats, indicating that vascular smooth muscle function was unaffected by fructose treatment. The 4-week treatment of the high fructose fed rats with naringin significantly restored $\mathrm{ACh}$ induced vasorelaxaton to the levels observed in the control rats but had no effect on SNP-induced relaxation.

As shown in Fig. 2, pre-incubation of aortic rings with indomethacin did not affect the vasodilator response to ACh in any group $\left(\mathrm{R}_{\max }\right.$ control $86 \pm 5$; fructose $81 \pm 5$; fructose+naringin $85 \pm 6 \%$ ), indicating that cyclo-oxygenase products, including prostacyclin, did not contribute to endothelium dependent relaxation. In the presence of L-NNA, ACh induced relaxation was completely abolished in aortic rings from the control rats,
Table I. Effect of oral administration (4 weeks) of naringin $(100 \mathrm{mg} / \mathrm{kg} / \mathrm{day})$ on body weight and blood parameters in fructose fed rats.

\begin{tabular}{lccc}
\hline Parameters & C & F & FN \\
\hline Initial body weight $(\mathrm{g})$ & $256 \pm 3$ & $250 \pm 3$ & $251 \pm 2$ \\
Final body weight $(\mathrm{g})$ & $487 \pm 7$ & $535 \pm 6$ & $483 \pm 6$ \\
Blood glucose $(\mathrm{mg} / \mathrm{dl})$ & $99 \pm 9$ & $149 \pm 13$ & $114 \pm 4$ \\
TC $(\mathrm{mg} / \mathrm{dl})$ & $60 \pm 7^{\mathrm{a}}$ & $78 \pm 5$ & $63 \pm 3^{\mathrm{a}}$ \\
TG $(\mathrm{mg} / \mathrm{dl})$ & $61 \pm 7^{\mathrm{a}}$ & $82 \pm 5$ & $52 \pm 4^{\mathrm{a}}$ \\
HDL-C (mg/dl) & $30 \pm 4$ & $26 \pm 2$ & $27 \pm 1$ \\
LDL-C (mg/dl) & $24 \pm 5^{\mathrm{a}}$ & $37 \pm 2$ & $26 \pm 3^{\mathrm{a}}$ \\
\hline
\end{tabular}

Values are expressed as mean \pm SEM $(n=8)$. ${ }^{\mathrm{a}} \mathrm{P}<0.05$ compared with F group. C, control rats; F, fructose-fed rats; FN, fructose fed rats treated naringin.
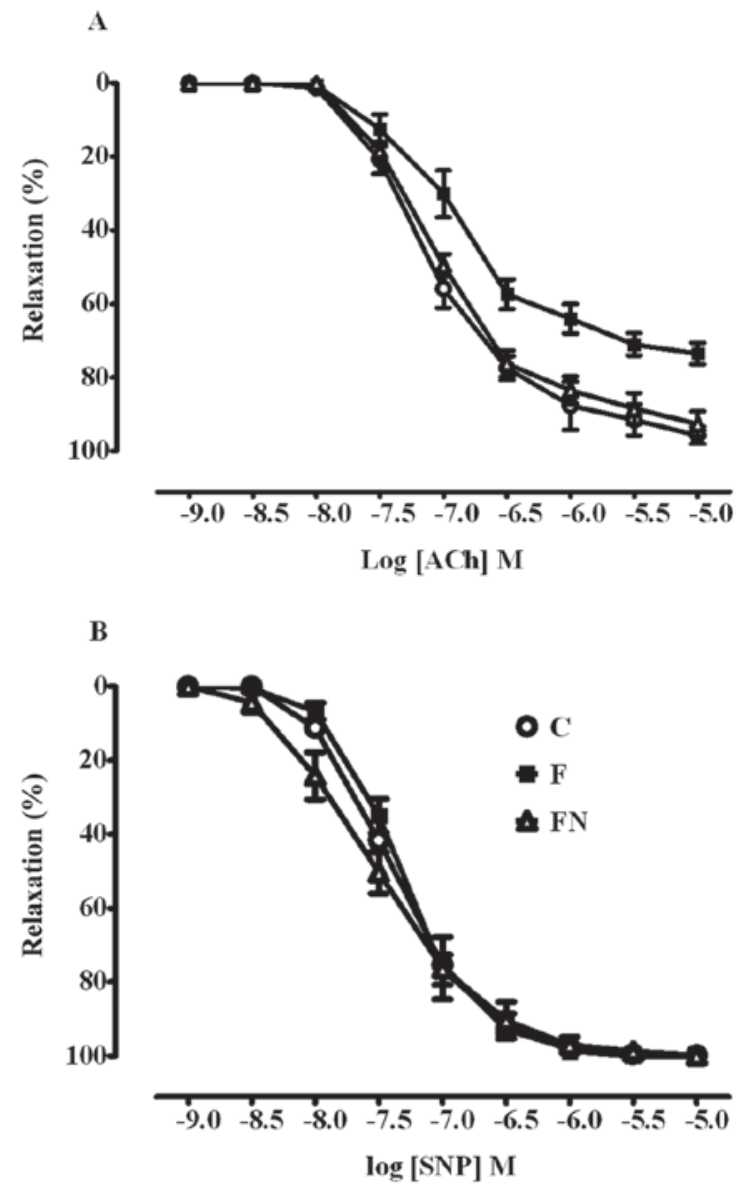

Figure 1. Effect of naringin treatment on concentration response curves to the (A) endothelium-dependent dilator $\mathrm{ACh}$ and the (B) endothelium-independent dilator SNP in aortic rings from fructose fed rats. In each group of experiments the aortic rings were precontracted to a similar level using PE (50 $\pm 2 \%$ of KPSS). ACh, acetylcholine; SNP, sodium nitroprusside; C, control rats; F, fructose-fed rats; FN, fructose fed rats treated naringin; PE, phenylephrine.

but was only partially inhibited in aortae from the fructose fed rats $\left(\mathrm{R}_{\max }\right.$ control 0 ; fructose $\left.18 \pm 2 \%, \mathrm{P}<0.01\right)$. In the $\mathrm{FN}$ groups, the relaxant response to ACh was almost completely 
Table II. Comparison of the sensitivity $\left(\mathrm{pEC}_{50}\right)$ and maximum response $\left(\mathrm{R}_{\max }\right)$ to ACh and SNP in aortic rings from control, fructose-fed rats, and fructose fed rats with naringin treatment (100 mg/kg/day).

\begin{tabular}{lccccc}
\hline & \multicolumn{2}{c}{$\mathrm{ACh}$} & & \multicolumn{2}{c}{$\mathrm{SNP}$} \\
\cline { 2 - 3 } \cline { 5 - 6 } Group & $\mathrm{pEC}_{50}$ & $\mathrm{R}_{\max }$ & & $\mathrm{pEC}_{50}$ & $\mathrm{R}_{\max }$ \\
\hline $\mathrm{C}$ & $7.17 \pm 0.09$ & $94 \pm 3$ & & $7.42 \pm 0.13$ & $100 \pm 0.1$ \\
$\mathrm{~F}$ & $6.91 \pm 0.08$ & $73 \pm 4^{\mathrm{a}}$ & & $7.23 \pm 0.07$ & $100 \pm 0.1$ \\
FN & $7.05 \pm 0.51$ & $95 \pm 3^{\mathrm{b}}$ & & $7.76 \pm 0.03$ & $100 \pm 0.2$ \\
\hline
\end{tabular}

All data are shown as the mean $\pm \operatorname{SEM}(n=8)$. ${ }^{\mathrm{P}}<0.01$ compared with $\mathrm{C}$ group (Dunnett's test); ${ }^{\mathrm{b}} \mathrm{P}<0.01$ compared with $\mathrm{F}$ group (Dunnett's test). ACh, acetylcholine; SNP, sodium nitroprusside; C, control rats; $\mathrm{F}$, fructose-fed rats; FN, fructose fed rats treated naringin.

inhibited by L-NNA ( $\mathrm{R}_{\max }$ fructose+naringin $7 \pm 2$; fructose $18 \pm 2 \%, \mathrm{P}<0.01)$, indicating that endothelium-derived NO plays an important role in the vascular effect of naringin. The combination of L-NNA plus indomethacin totally abolished the response to $\mathrm{ACh}$ in aortic rings of all rats.

Effect on NO levels. Serum NOx levels were measured to estimate the nitric oxide bioactivity. After 8 weeks, the serum NOx levels of the fructose fed rats were significantly lower than that of the control rats (control 0.26 \pm 0.03 ; fructose $0.13 \pm 0.02 \mu \mathrm{M} / \mathrm{ml}, \mathrm{P}<0.01)$. However, naringin treatment of the fructose fed rats significantly increased NOx levels compared to the fructose fed group (Group 2, the F group), (fructose+naringin $0.21 \pm 0.01$; fructose $0.13 \pm .0 .02 \mu \mathrm{M} / \mathrm{ml}$, $\mathrm{P}<0.01)$.

Effect of eNOS, p-eNOS, and nitrotyrosine expression in aortic rings. The fructose fed rats (the $\mathrm{F}$ group) exhibited a significant decrease in protein expressions of eNOS and phosphorylation of eNOS at Ser1177 in the aortic tissues compared to the control rats. Treatment of the fructose fed rats with naringin (the FN group) significantly increased aortic eNOS and phosphorylation of eNOS expression. In addition, fructose feeding significantly increased the expression of nitrotyrosine in aortic tissues, but this was reversed by treatment with naringin (Fig. 3).

\section{Discussion}

The present study demonstrated that treatment of fructose fed rats with naringin $(100 \mathrm{mg} / \mathrm{kg} / \mathrm{d})$ for 4 weeks improved the impaired endothelial dependent relaxation in aortic rings and increased serum NO level. These effects of naringin may be associated with enhanced aortic eNOS and p-eNOS expression, and reduced expression of nitrotyrosine in aortic tissues of fructose fed rats.

The model of fructose-drinking rats has been widely reported to develop metabolic abnormalities, including hypertriglyceridemia, hyperglycemia, hyperinsulinemia and obesity, as well as endothelial dysfunction $(1,11,12,18)$. These abnormalities are closely associated with the development of
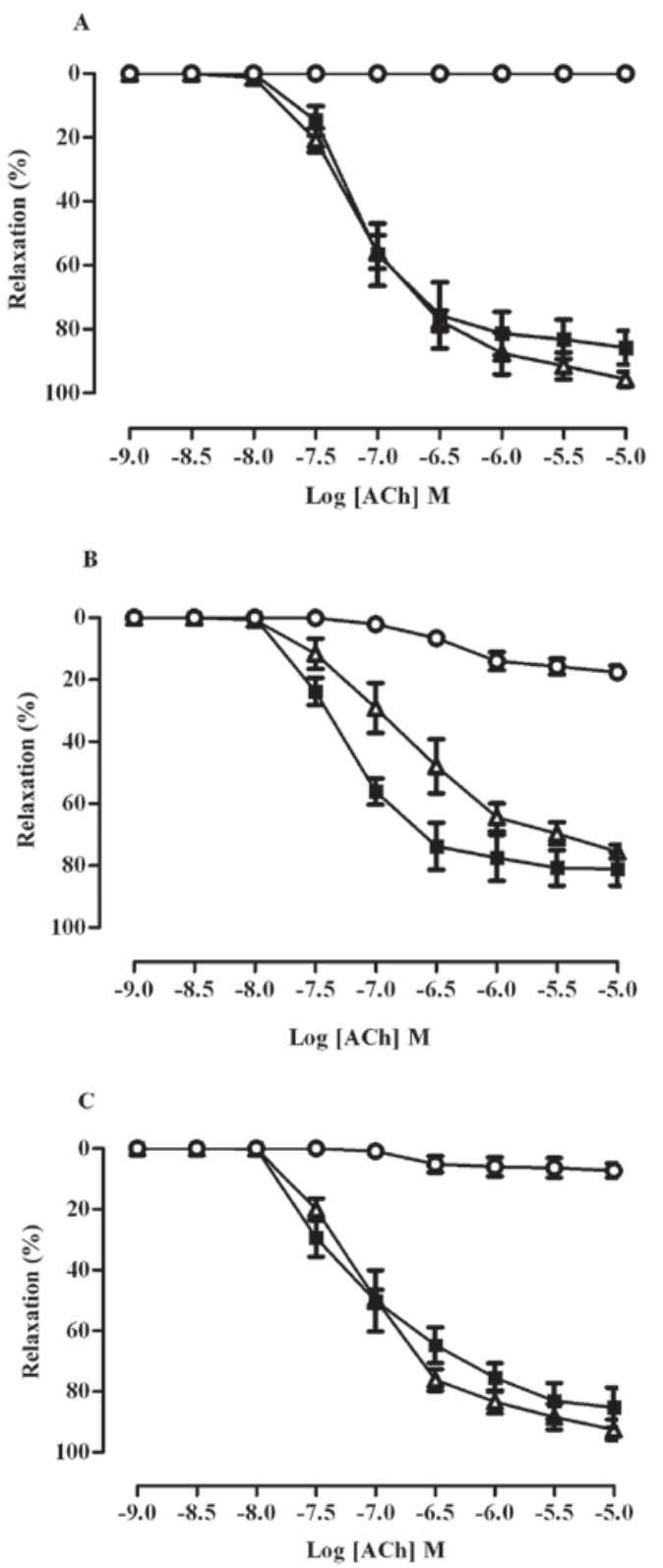

Figure 2. Concentration-response curves for acetylcholine in phenylephrine-contracted aortic rings, alone $(\cdot)$ or in the presence of indomethacin $(\Delta)$ or NG-nitro-l-arginine (1-NNA; o) in (A) C group, (B) F group and (C) FN group. All data are shown as the mean \pm SEM. ACh, acetylcholine; $\mathrm{C}$, control rats; F, fructose-fed rats; FN, fructose fed rats treated naringin; PE, phenylephrine.

CVD (3-5,9). However, the concentration of fructose used in our experiment and in most animal studies was higher than that consumed by human, thereby increasing significant metabolic changes $(1,28-30)$. This study demonstrated that rats given $10 \%$ fructose in drinking water for 12 weeks, exhibited increased blood glucose and developed dyslipidemia, as indicated by the elevated levels of serum TC, LDL-C and TG. Oral administration of naringin to rats for 4 weeks ameliorated high fructose-induced hyperglycemia and hyperlipidemia.

There is evidence to support the beneficial effect of naringin on these metabolic alterations in animals. Previous studies have shown that naringin reduced blood glucose and lipid levels in several animal models of diabetes and diet-induced metabolic syndromes $(19,20,22,31)$. In type 2 diabetic $d b / d b$ 

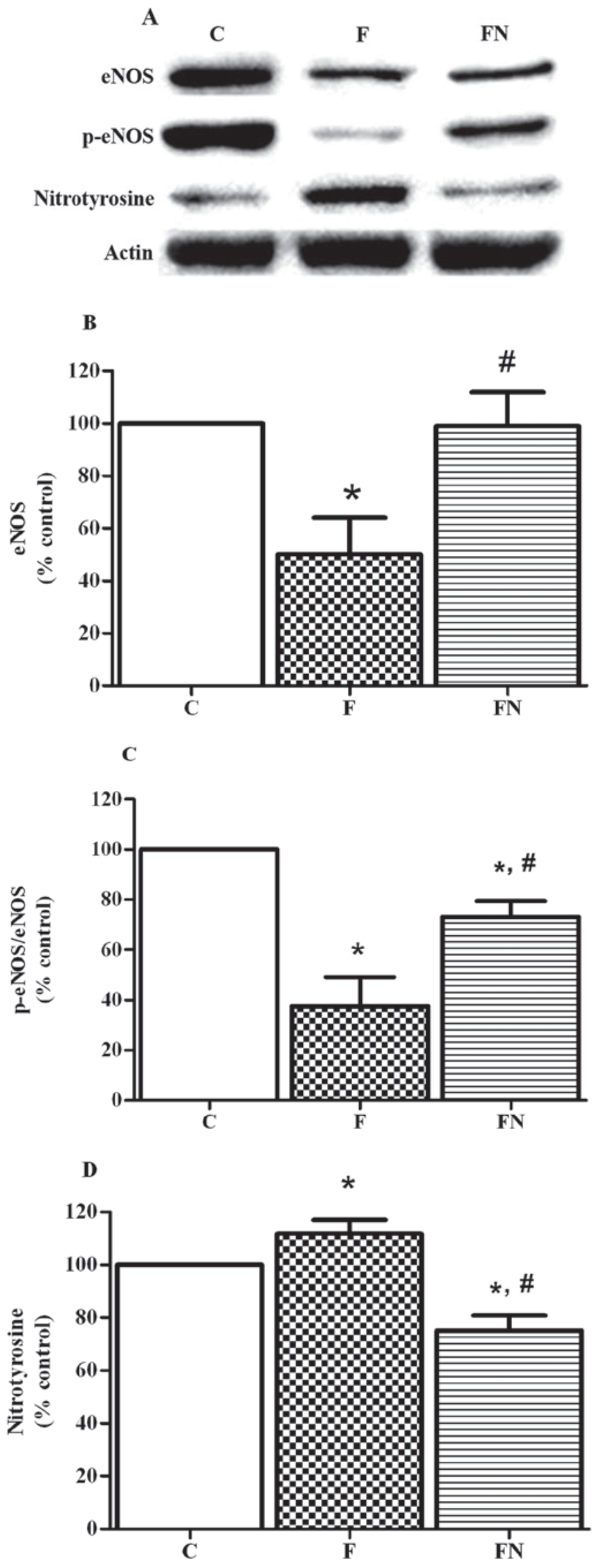

Figure 3. Protein expression of (B) eNOS, (C) phosphorylated eNOS at Ser1177 residue, and (D) nitrotyrosine in aortic tissues from control (C), fructose (F), and fructose plus naringin (FN) groups. (A) Representative western blottings. Results are expressed as mean \pm SEM ( $n=6$ per group) and presented as percentage of control value. ${ }^{*} \mathrm{P}<0.05$ compared with control group, ${ }^{\#} \mathrm{P}<0.05$ compared with F group. eNOS, endothelial nitric oxide synthase. mice, naringin lowered hyperlipidemia and hyperglycemia through regulating the lipid metabolism and affecting the gene expression of glucose-regulating enzymes (32). In mice fed high fat diet, naringin could activate AMPK-mediated MAPKs signaling pathway, resulting in the reduction of insulin resistance, hyperglycemia, and hyperlipidemia (22).

Both dyslipidemia and hyperglycemia have been widely reported to be a leading cause of vascular endothelial dysfunction, which is an early marker of atherosclerosis and cardiovascular disease $(33,34)$. Endothelial dysfunction is generally characterized by a decrease in endothelial dependent relaxation. The vascular endothelium plays a vital role in the regulation of basal vascular tone through the synthesis and release of several vasodilators including nitric oxide (NO), prostaglandin $\mathrm{I}_{2}\left(\mathrm{PGI}_{2}\right)$ and endothelium-derived hyperpolarizing factor (EDHF) (7). It has been reported that endothelium-derived NO is a predominant mediator of endothelial-dependent relaxation in aortae, and its bioavailability is impaired in several pathophysiological states such as hyperlipidemia, metabolic syndrome, diabetes and hypertension $(35,36)$. There is increasing evidence that high fructose induced endothelial dysfunction is associated with the decreased NO bioavailability in the vasculature $(8,12)$.

This study demonstrated that the vasorelaxation response to ACh but not SNP was decreased in aortae from fructose fed rats, indicating that fructose feeding caused an impairment of endothelium dependent relaxation in rat aortae. These findings are consistent with previous reports $(10,17)$, which demonstrated that an impaired endothelium function was found in aortic rings of fructose fed animals. The presence of NOS inhibitor L-NNA partially inhibited ACh-induced relaxation in aortic rings of fructose fed rats, indicating that fructose feeding impaired the contribution of NO to endothelium-dependent relaxation in aortic tissues. An impaired endothelium derived NO was also confirmed by reduced serum nitrite/nitrate concentration in fructose fed rats.

Previous studies have reported that naringin treatment improved endothelial dysfunction in stroke-prone spontaneously hypertensive rats (25) and high carbohydrate, high fat diet-fed rats (21). In this study, the treatment of fructose fed rats with naringin for 4 weeks restored ACh-induced relaxation in aortae to levels similar to those observed in control rats (Fig. 1 and Table II). The possibility that naringin improved endothelium dependent relaxation by increasing NO bioavailability was investigated. This study demonstrated that the relaxation to ACh was totally abolished in the presence of L-NNA but unaffected by indomethacin (Fig. 2). We also found that naringin increased serum nitrite/nitrate levels in fructose fed rats. These results indicate that the beneficial effects of naringin on endothelial dysfunction in fructose fed rats are due to its ability to restore endothelial derived NO.

Possible mechanisms for maintaining NO bioavailability and improving endothelium dependent relaxation in fructose fed rats by naringin treatment may be related to an increase in NO production and/or decrease in NO degradation by ROS. The production of NO is regulated by eNOS activity, which is activated by phosphorylation at an activation site such as Ser1177, Ser633 and Ser614 (37,38). There is evidence that phosphorylation of eNOS at serine 1117 is a crucial target for intervention to improve endothelial dysfunction (15). In 
addition, there is growing evidence that an overproduction of ROS, which is generally generated by a cellular disturbance in glucose or/and lipid metabolism leads to the degradation of NO (39-41). Superoxide rapidly reacts with NO to form the powerful oxidant peroxynitrite which causes the nitration of proteins leading to the impairing of the function of cellular proteins including eNOS protein (15). Therefore, in our study, nitrotyrosine was detected to indirectly indicate ROS mediated NO inactivation and peroxynitrite formation. It has been reported that fructose-fed rats exhibited a decreased expression of eNOS and p-eNOS in several tissues including aorta $(8,42,43)$. In addition fructose has been demonstrated to generate peroxynitrite through increased superoxide production and enhanced methyglyoxal formation (16).

Consistent with the impairment of endothelium dependent relaxation and the decrease in NO levels, it was found that the reduction of expression of eNOS and p-eNOS, and the elevation of nitrotyrosine expression in fructose fed rats. Previous studies demonstrated that naringin elevated expression of p-eNOS (Ser1177) and reduced expression of nitrotyrosine in myocardial ischemia reperfusion injury (44). In this study, it was found that oral administration of naringin to fructose fed rats for 4 weeks increased the expression of eNOS and p-eNOS (Ser1177), and decreased the expression of nitrotyrosine in aortic tissues (Fig. 3). These results suggest that naringin treatment increases NO bioavailability through enhanced eNOS activity and attenuated NO inactivation to nitrotyrosine, resulting in the improved endothelial dysfunction in fructose fed rats. However, the mechanisms of increased eNOS and p-eNOS expression in naringin and fructose-fed rats were still unclear and further study is needed to clarify the mechanisms underlying the upregulated eNOS and p-eNOS protein expression.

In conclusion, the present study suggests that treatment with naringin improves endothelial function in the aortic rings of fructose fed rats. The vascular effect of naringin may be, in part, due to improving NO bioavailability, increasing eNOS activity, and preventing the generation of peroxynitrite.

\section{Acknowledgements}

This study was supported by grants from the National Research Council of Thailand. The authors would like to thank Mr. Roy Morien of the naresuan university language centre for his editing assistance and advice on english expression in this document. The authors declare no conflict of interest.

\section{References}

1. Tappy L, Lê KA, Tran C and Paquot N: Fructose and metabolic diseases: New findings, new questions. Nutrition 26: 1044-1049, 2010.

2. Johnson RJ, Segal MS, Sautin Y, Nakagawa T, Feig DI, Kang DH, Gersch MS, Benner S and Sánchez-Lozada LG: Potential role of sugar (fructose) in the epidemic of hypertension, obesity and the metabolic syndrome, diabetes, kidney disease, and cardiovascular disease. Am J Clin Nutr 86: 899-906, 2007.

3. Nelson RH: Hyperlipidemia as a risk factor for cardiovascular disease. Prim Care 40: 195-211, 2013.

4. Lorber D: Importance of cardiovascular disease risk management in patients with type 2 diabetes mellitus. Diabetes Metab Syndr Obes 7: 169-183, 2014.
5. Favero G, Paganelli C, Buffoli B, Rodella LF and Rezzani R: Endothelium and its alterations in cardiovascular diseases: Life style intervention. Biomed Res Int 2014: 801896, 2014.

6. Fadel PJ: Nitric oxide and cardiovascular regulation: Beyond the endothelium. Hypertension 69: 778-779, 2017.

7. Vanhoutte PM, Shimokawa H, Feletou M and Tang EH: Endothelial dysfunction and vascular disease-a 30th anniversary update. Acta Physiol (Oxf) 219: 22-96, 2017.

8. Pektaş MB, Sadi G and Akar F: Long-term dietary fructose causes gender-different metabolic and vascular dysfunction in rats: Modulatory effects of resveratrol. Cell Physiol Biochem 37: 1407-1420, 2015.

9. Jia G, Aroor AR, Whaley-Connell AT and Sowers JR: Fructose and uric acid: Is there a role in endothelial function? Curr Hypertens Rep 16: 434, 2014.

10. Kho MC, Lee YJ, Cha JD, Choi KM, Kang DG and Lee HS: Gastrodia elata ameliorates high-fructose diet-induced lipid metabolism and endothelial dysfunction. Evid Based Complement Alternat Med 2014: 101624, 2014.

11. Tran LT, Yuen VG and McNeill JH: The fructose-fed rat: A review on the mechanisms of fructose-induced insulin resistance and hypertension. Mol Cell Biochem 332: 145-159, 2009.

12. El-Bassossy H, Badawy D, Neamatallah T and Fahmy A: Ferulic acid, a natural polyphenol, alleviates insulin resistance and hypertension in fructose fed rats: Effect on endothelial-dependent relaxation. Chem Biol Interact 254: 191-197, 2016.

13. Li T, Lu X, Sun Y and Yang X: Effects of spinach nitrate on insulin resistance, endothelial dysfunction markers and inflammation in mice with high-fat and high-fructose consumption. Food Nutr Res 60: 32010, 2016.

14. Nade VS, Kawale LA and Patel KM: Protective effect of sitagliptin and rosuvastatin combination on vascular endothelial dysfunction in type-2 diabetes. Indian J Pharm Sci 77: 96-102, 2015.

15. Zhao Y, Vanhoutte PM and Leung SW: Vascular nitric oxide: Beyond eNOS. J Pharmacol Sci 129: 83-94, 2015.

16. Wang H, Meng QH, Chang T and Wu L: Fructose-induced peroxynitrite production is mediated by methylglyoxal in vascular smooth muscle cells. Life Sci 79: 2448-2454, 2006.

17. Babacanoglu C, Yildirim N, Sadi G, Pektas MB and Akar F: Resveratrol prevents high-fructose corn syrup-induced vascular insulin resistance and dysfunction in rats. Food Chem Toxicol 60: 160-167, 2013

18. Litterio MC, Vazquez Prieto MA, Adamo AM, Elesgaray R, Oteiza PI, Galleano M and Fraga CG: (-)-Epicatechin reduces blood pressure increase in high-fructose-fed rats: Effects on the determinants of nitric oxide bioavailability. J Nutr Biochem 26: 745-751, 2015.

19. Chanet A, Milenkovic D, Deval C, Potier M, Constans J, Mazur A, Bennetau-Pelissero C, Morand C and Bérard AM: Naringin, the major grapefruit flavonoid, specifically affects atherosclerosis development in diet-induced hypercholesterolemia in mice. J Nutr Biochem 23: 469-477, 2012.

20. Adebiyi OA, Adebiyi OO and Owira PM: Naringin reduces hyperglycemia-induced cardiac fibrosis by relieving oxidative stress. PLoS One 11: e0149890, 2016.

21. Alam MA, Kauter K and Brown L: Naringin improves diet-induced cardiovascular dysfunction and obesity in high carbohydrate, high fat diet-fed rats. Nutrients 5: 637-650, 2013.

22. Pu P, Gao DM, Mohamed S, Chen J, Zhang J, Zhou XY, Zhou NJ, Xie J and Jiang H: Naringin ameliorates metabolic syndrome by activating AMP-activated protein kinase in mice fed a high-fat diet. Arch Biochem Biophys 518: 61-70, 2012.

23. Sharma AK, Bharti S, Ojha S, Bhatia J, Kumar N, Ray R, Kumari S and Arya DS: Up-regulation of PPAR $\gamma$, heat shock protein-27 and -72 by naringin attenuates insulin resistance, $\beta$-cell dysfunction, hepatic steatosis and kidney damage in a rat model of type 2 diabetes. Br J Nutr 106: 1713-1723, 2011.

24. Li W, Wang C, Peng J, Liang J, Jin Y, Liu Q, Meng Q, Liu K and Sun $\mathrm{H}$ : Naringin inhibits TNF- $\alpha$ induced oxidative stress and inflammatory response in HUVECs via Nox4/NF- $\mathrm{B}$ and PI3K/Akt pathways. Curr Pharm Biotechnol 15: 1173-1182, 2014.

25. Ikemura M, Sasaki Y, Giddings JC and Yamamoto J: Preventive effects of hesperidin, glucosyl hesperidin and naringin on hypertension and cerebral thrombosis in stroke-prone spontaneously hypertensive rats. Phytother Res 26: 1272-1277, 2012.

26. Friedewald WT, Levy RI and Fredrickson DS: Estimation of the concentration of low-density lipoprotein cholesterol in plasma, without use of the preparative ultracentrifuge. Clin Chem 18: 499-502, 1972. 
27. Ali SF, Nguyen JC, Jenkins TA and Woodman OL: Tocotrienol-rich tocomin attenuates oxidative stress and improves endothelium-dependent relaxation in aortae from rats fed a high-fat western diet. Front Cardiovasc Med 3: 39, 2016.

28. Ventura EE, Davis JN and Goran MI: Sugar content of popular sweetened beverages based on objective laboratory analysis: Focus on fructose content. Obesity (Silver Spring) 19: 868-874, 2011.

29. Toop CR and Gentili S: Fructose beverage consumption induces a metabolic syndrome phenotype in the rat: A systematic review and meta-analysis. Nutrients 8: pii: E577, 2016

30. Zhang DM, Jiao RQ and Kong LD: High dietary fructose: Direct or indirect dangerous factors disturbing tissue and organ functions. Nutrients 9: pii: E335, 2017.

31. Mahmoud AM, Ashour MB, Abdel-Moneim A and Ahmed OM: Hesperidin and naringin attenuate hyperglycemia-mediated oxidative stress and proinflammatory cytokine production in high fat fed/streptozotocin-induced type 2 diabetic rats. J Diabetes Complications 26: 483-490, 2012.

32. Jung UJ, Lee MK, Park YB, Kang MA and Choi MS: Effect of citrus flavonoids on lipid metabolism and glucose-regulating enzyme mRNA levels in type-2 diabetic mice. Int J Biochem Cell Biol 38: 1134-1145, 2006

33. Tabit CE, Chung WB, Hamburg NM and Vita JA: Endothelial dysfunction in diabetes mellitus: Molecular mechanisms and clinical implications. Rev Endocr Metab Disord 11: 61-74, 2010.

34. Huang Q, QinL, DaiS,Zhang H,PasulaS,ZhouH,ChenHand Min W: AIP1 suppresses atherosclerosis by limiting hyperlipidemia-induced inflammation and vascular endothelial dysfunction. Arterioscler Thromb Vasc Biol 33: 795-804, 2013.

35. Widmer RJ and Lerman A: Endothelial dysfunction and cardiovascular disease. Glob Cardiol Sci Pract 2014: 291-308, 2014.

36. Wong WT, Wong SL, Tian XY and Huang Y: Endothelial dysfunction: The common consequence in diabetes and hypertension. J Cardiovasc Pharmacol 55: 300-307, 2010.
37. Yu L, Liu Y, Qiu Z, Liu S, Gao X and Zhu D: Cellular mechanisms and intracellular signaling pathways for the modulation of eNOS in pulmonary arteries by 15 -HETE. J Recept Signal Transduct Res 32: 87-95, 2012.

38. Li S, Li Q, Lv X, Liao L, Yang W, Li S, Lu P and Zhu D: Aurantio-obtusin relaxes systemic arteries through endothelial PI3K/AKT/eNOS-dependent signaling pathway in rats. J Pharmacol Sci 128: 108-115, 2015.

39. Steven S, Daiber A, Dopheide JF, Münzel T and Espinola-Klein C: Peripheral artery disease, redox signaling, oxidative stress-Basic and clinical aspects. Redox Biol 12: 787-797, 2017.

40. Lau YS, Tian XY, Huang Y, Murugan D, Achike FI and Mustafa MR: Boldine protects endothelial function in hyperglycemia-induced oxidative stress through an antioxidant mechanism. Biochem Pharmacol 85: 367-375, 2013.

41. Chtourou Y, Slima AB, Makni M, Gdoura R and Fetoui H: Naringenin protects cardiac hypercholesterolemia-induced oxidative stress and subsequent necroptosis in rats. Pharmacol Rep 67: 1090-1097, 2015.

42. Stanišić J, Korićanac G, Ćulafić T, Romić S, Stojiljković M, Kostić M, Pantelić M and Tepavčević S: Low intensity exercise prevents disturbances in rat cardiac insulin signaling and endothelial nitric oxide synthase induced by high fructose diet. Mol Cell Endocrinol 420: 97-104, 2016.

43. Xu X, Tu L, Wang L, Fang X and Wang DW: CYP2J3 gene delivery reduces insulin resistance via upregulation of eNOS in fructose-treated rats. Cardiovasc Diabetol 10: 114, 2011.

44. Rani N, Bharti S, Manchanda M, Nag TC, Ray R, Chauhan SS, Kumari S and Arya DS: Regulation of heat shock proteins 27 and 70, p-Akt/p-eNOS and MAPKs by Naringin Dampens myocardial injury and dysfunction in vivo after ischemia/reperfusion. PLoS One 8: e82577, 2013. 